This is the accepted version of the article:

Marquez S., Álvarez M., Fariña Santana D., Homs-Corbera A., Domínguez C., Lechuga L. M.. Array of Microfluidic Beam Resonators for Density and Viscosity Analysis of Liquids. Journal of Microelectromechanical Systems, (2017). 26. 7945266: 749 - . 10.1109/J MEMS.2017.2709944.

Available at: https://dx.doi.org/10.1109/J MEMS.2017.2709944 


\title{
Array of Microfluidic Beam Resonators for Density and Viscosity Analysis of Liquids
}

\author{
S. Marquez, M. Alvarez, D. Fariña, A Homs-Corbera, C. Dominguez and L.M Lechuga Member, \\ IEEE
}

Abstract-This paper reports on the design, fabrication an 40 evaluation of a mass density and viscosity sensor based on an arra 1 of polysilicon microbeam resonators integrated with $20 \mathrm{pL}$ fluidiq 2 microchannels. When filled with water, resonators exhibit resonant 3 frequencies close to $500 \mathrm{KHz}$ and Q-factor values of 400 operating at atmospheric pressure and ambient temperature. Real-time measurements are highly reproducible and only require $250 \mu \mathrm{L}$ of the sample fluid. The built-in interferometric readout enable 6 automatic detection of the beams, increasing the throughput 7 analysis and reducing detection times. The frequency shift respons $\$ 8$ shows a linear behavior in accordance with the density of evaluated 9 solvents, organic solutions and alcoholic drinks, reporting a mass responsivity of $7.4 \mathrm{~Hz} / \mathrm{pg}$. Also, the sensor is capable of measuring the viscosity of liquid phase samples with a resolution of $0.15 \mathrm{cP}$ by 1 tracking the Q-factor response of the sensor within a linear regim 52 between $1 \mathrm{cP}$ to $2.6 \mathrm{cP}$. This approach demonstrates the ability t53 identify in real-time changes of fluids in the liquid phase that couls 4 provide a valuable assessment for bioanalytical applications.

Index Terms-Array, density, liquids, microfluidic channels, $Q_{\overline{5}}^{56}$ factor, resonators, resonant frequency, viscosity.

\section{INTRODUCTION}

DENTIFICATION of changes in fluid properties of samples 26 is essential for a wide range of industrial and medical applications. Density and rheological properties of fluids, such as viscosity, are employed to detect food quality [1]-[2], DNA4 solutions [3] and even single cells [4]. However, current analytical methods generally require large volume samples, need long evaluation times and have a limited sensitivity range [5],[6] Over the last decade, microelectromechanical sensors (MEMS have become one of the most promising tools for the achievement of high sensitive sensors due to their miniature size low mass, and compatibility with CMOS (complementary metaloxide semiconductor) technologies [7],[8]. The scope of demonstrated applications of MEMS devices for density and rheological parameters of fluids includes: viscoelastic properties

Manuscript received December 23, 2016. This work was supported in part by the National Council for Science and Technology (CONACyT-Mexico) and the ICTS IMB-CNM (CSIC) clean-room facilities under project NGG-244. The nanoB2A is a consolidated research group (Grup de Recerca) of the Generalitat de Catalunya and has support from the Departament d'Universitats, Recerca i Societat de la Informació de la Generalitat de Catalunya (2014 SGR 624). ICN2 is the recipient of Grant SEV-2013-0295 from the "Severo Ochoa Centers of Excellence" Program of Spanish MINECO.

S. Marquez, D. Fariña, A Homs-Corbera and L.M Lechuga are with Nanobiosensors and Bioanalytical Applications Group, Catalan Institute of of blood and plasma coagulation [10],[11], viscosity measurements of hydrocarbons, silicone oils, oil/fuel mixtures [11]-[13] and gases [14], characterization of polymer solutions [15], concentration of sugar mixtures [16] and ethanol solutions [1],[17], among others. However, when a MEMS resonator is immersed in a viscous fluid, the overdamped response of the resonator produces a rapid dissipation of energy degrading the sensitivity of the sensor with respect to the viscosity of the medium.

An innovative approach to solve this limitation was proposed by Burg et al. [18]. This approach involved integrating a fluidic channel into a suspended microcantilever, namely suspended microchannel resonator (SMR), thereby avoiding damping and viscous drag produced by the fluid environment. Since then, various solutions have been proposed to improve the sensitivity of SMR devices either by decreasing their effective mass or by proposing different materials and designs on their geometry. For example, Khan et. al [13] used silicon-rich nitride (SRN) as structural material to build transparent microchannels that facilitated the visual inspection of processes taking place inside the channels. This approach further derived density and viscosity measurements in liquid phase reagents. Suspended resonators have also been fabricated based on embedded microchannels in plate Lamé resonators to exhibit higher frequency responses and Q-factor values without the need for vacuum and packaging [19]. Another solution reported the fabrication of a suspended doubly clamped beam sensor at nanoscale dimensions with the aim of reducing the effective mass of the resonator [20]. Despite these promising advantages, reducing the mass and size of resonators in order to achieve sensitive transducers complicates the fabrication process. Furthermore, kinetics plays an important role in these type of devices due to the maximum volume of fluid that can flow through the microchannels and the maximum achievable flow rates to optimize the detection times [21]. Whereas a single SMR device is highly sensitive and suitable for identifying individual reagents, a device approach that includes

Nanoscience and Nanotechnology (ICN2) CSIC, The Barcelona Institute of Science and Technology, Campus UAB, Bellaterra, 08193 Barcelona, Spain and with the CIBER-BBN Networking Center on Bioengineering, Biomaterials and Nanomedicine, Spain (e-mail: salomon.marquez@icn2.cat; dfarinas@cin2.es; antoni.homs@icn.cat; laura.lechuga@icn2.cat)

M. Alvarez and C. Dominguez are with the Microelectronics Institute of Barcelona (IMB-CNM), CSIC, Barcelona, Spain (e-mail: mar.alvarez@imbcnm.csic.es; Carlos.Dominguez@imb-cnm.csic.es)

This paper has supplementary downloadable material that consists of a video. 

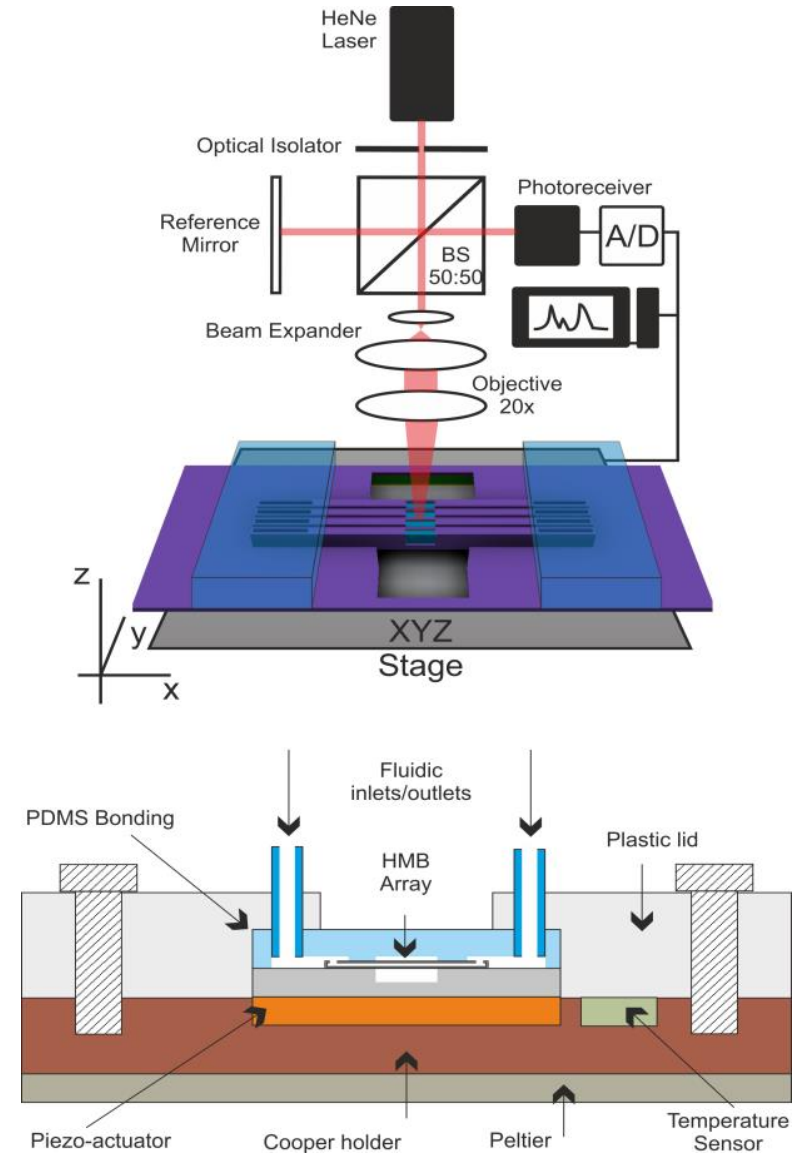

Fig. 1 (Top) Schematic view of the HMB sensor and the experimental setup 48 A custom free space interferometer acquires the driven excitation response of 49 the resonators while a sample solution is streaming through the embedded 50 microchannels. During experiments, temperature was fixed at $23^{\circ} \mathrm{C} \pm 0.1$. 51 (Bottom) Details of the copper fixture housing the Peltier module and the 51 piezo-ceramic actuator for driving the excitation of the resonators.

1 an array of resonators with single inlet/outlet embedded microchannels can constitute an efficient sensing platform for reducing sample evaluation times. Besides, this new approach can be used for the simultaneous detection of different samples 58 in real-time.

Therefore, in our approach, we have developed a mass density 59 and viscosity sensor for the real-time identification of Newtonia 0 fluids in the liquid phase using an array of suspended polysilicon microbeams, namely Hollow Microbeam (HMB) resonators (see 1 Fig. 1). The sensor consists of four closely spaced doubl 62 clamped beams with embedded microfluidic channels whereiø 3 each microchannel has a volume of around $20 \mathrm{pL}$. The solutio64 drawn up in our work includes four main contribution $\$ 95$ fabrication of a sensor array of resonators with embedde $\$ 6$ microchannels, implementation of an experimental readout for automatic detection, increase of the precision of rheologicab 7 measurements of liquids, and usage of polysilicon as main structural material of the sensor. As proof of concept, we hav 68 studied the sensor performance in a flow-through detection mod69 to measure density and viscosity of different solvents and0 organic solutions. Also, we have analyzed commercial alcoholiđ 1 beverages demonstrating that the HMB resonators can reduce to2 res

$$
\text { micror }
$$
microchannel, and $w_{b}$ and $h_{b}$, the width and height of the beam, respectively. The total effective mass of the resonator is a contribution of not only the structural material but also of the added mass induced by the sample fluid. For this reason, we modeled the resonators as a multimorph doubly clamped beams formed by equal length layers in which the effective mass is defined as [23]:

$$
m_{b}=\frac{26}{70} L \sum_{i=1}^{n} \rho_{i} A_{i}
$$

where $\rho_{\mathrm{i}}$ is the density of the layer "i" with its corresponding cross-sectional area $\mathrm{A}_{\mathrm{i}}$. By substituting the lump-parameter definitions of $\mathrm{k}_{\mathrm{b}}$ and $\mathrm{m}_{\mathrm{b}}$ into (1), the approximated fundamental resonant frequency of the HMB resonator is:

$$
f_{r, b}=\frac{22.736 h_{b}}{2 \pi L^{2}} \sqrt{\frac{E}{12\left[\alpha \rho_{f}+(1-\alpha) \rho_{b}\right]}}
$$

where $\rho_{b}$ and $\rho_{f}$ are the structural material and sample fluid densities, respectively. The parameter $\alpha$ is the ratio of the microfluidic channel surface to the beam cross-sectional area defined as $\alpha=w_{f} h_{f} / w_{b} h_{b}$. By monitoring the HMB resonant 
1 frequency response, we correlated the frequency changes as $\mathbf{5 4}$

2 function of the sample fluid density.

The quality factor $(\mathrm{Q})$ defines the ratio of stored energy to th 56 dissipated energy on each oscillation cycle of a resonator. O67 5 SMR devices, the dissipation of energy is mainly attributed t68 6 shearing of the contained fluid that is able to freely move insid69 7 the channel. Besides, as damping is a non-monotonic function 060 8 the sample viscosity inside of SMR resonators [24], the Q-factor 1 9 value can increase or decrease with respect to sample viscosit 22

10 [25]. Since the embedded microchannel is centered about th63 11 beam neutral axis in our approach, the magnitude of the shearing 4 12 effect can be determined by the dimensionless frequency number 5 $13 \beta=\rho_{\text {fluid }} \omega \mathrm{h}^{2}$ fluid $/ \mu$, where $\mathrm{h}_{\text {fluid }}$ is the channel height, $\mu$ is th66

dynamic viscosity, $\rho_{\text {fluid }}$ is the fluid density and $\omega$ is the resonan $(7$ frequency, respectively. Sader et al. [25] derived a non68 monotonic function $F(\beta)$ to understand the effect of the energ 69 dissipation of SMR devices as a function of the $\beta$ number. Fo70 the fundamental frequency mode of resonators, the non 71 monotonic function $F(\beta) \approx 0.152 \sqrt{ } \beta+38.7 / \beta[24]$ defines tw ${ }^{2}$ flow transition regimes at its minimum $(\beta=46)$. For $\beta<46$, there 3 is a low-inertia regime whereas for $\beta>46$, fluid inertia dominates. The dimensionless function $F(\beta)$ yields a maximum error estimation of $13 \%$ for all $\beta$. According to the fabricated dimensions of our resonators and the used liquid samples, lowinertia regime dominates in the HMB devices. By tracking the quality factor of the resonators while streaming different liquid samples, we analyzed the sensor response to viscosity changes.

Another important design parameter of the HMB resonators is related to the limits of pressure at both ends of the microchannel inlets in order to avoid the collapsing of the inner channel walls. A reasonable approximation with less than $10 \%$ error, for aspect ratios of $h / w \leq 0.7$, introduces a pressure difference of [26]:

$$
\Delta P\left[1-\frac{6\left(2^{5}\right)}{\pi^{5}} \frac{h_{f}}{w_{f}}\right]=\frac{12 \mu L}{w h_{f}^{3}} Q_{\text {flow }}
$$

where $\Delta \mathrm{P}$ is the pressure difference along the microfluidic channel; $\mu$ is the water viscosity $(1 \mathrm{cP})$ and $\mathrm{Q}_{\text {flow }}$ defines the flow rate $(\mu \mathrm{L} / \mathrm{min})$, respectively. According to this relationship, we can theoretically inject solutions at a pressure difference of up to $7 \mathrm{MPa}$, yielding flow rates of up to $45 \mu \mathrm{l} / \mathrm{min}$.

\section{FABRICATION AND CHARACTERIZATION}

\section{A. Device Design and Fabrication}

Four embedded microfluidic channels of $1300 \mu \mathrm{m}$ in length and cross-sectional area of $20 \mu \mathrm{m} \times 4 \mu \mathrm{m}$ integrate the array of nominally identical resonators, with a distance between each other of $13 \mu \mathrm{m}$, as Fig. 2 shows. This design establishes very short separation distances between the hollow doubly clamped beams so that the response of the sensors can be acquired by the optical readout system. The microchannel wall thickness is 1 $\mu \mathrm{m}$. The effective length of each resonator (about $275 \mu \mathrm{m}$ ) is set at each clamped side of a rectangular trench of $275 \mu \mathrm{m} \times 7500$ $\mu \mathrm{m}$. Fluidic inlets of $10 \mu \mathrm{m} \times 100 \mu \mathrm{m}$ in dimension are located at both end sides of the microchannels. To allow fluid exchange inside of the embedded microchannels, two polydimethylsiloxane (PDMS) delivery channels of crosssectional area of $200 \mu \mathrm{m} \times 35 \mu \mathrm{m}$ and length of $1.5 \mathrm{~mm}$ are integrated on both sides of the inlets of HMB resonators. The serpentine design of the PDMS fluid delivery channels is done in order to facilitate the visual inspection of the meniscus of the liquids during the filling step of the HMB sensors.

Hollow microbridges were fabricated using 4-in, type $\mathrm{p} \mathrm{silicon}$ wafers of $500 \mu \mathrm{m}$ thickness. Polysilicon was used as structural layer and boron phosphorus doped silicon oxide (BPSG) as sacrificial layer because of its high selectivity to polysilicon and high etching rate with HF $49 \%$. Thereafter, a $1 \mu \mathrm{m}$ layer of polysilicon was deposited by low-pressure chemical vapor deposition (LPCVD) at $580^{\circ} \mathrm{C}$ and $380 \mathrm{mtorr}$. The $4 \mu \mathrm{m}$ topology of microfluidic channels was patterned by reactive ion etching and hard contact photolithography. As follows, another layer of $1 \mu \mathrm{m}$ polysilicon was coated in order to enclose the microchannels. Access holes, located at both ends of the structures were etched on top of the polysilicon layer in order to

a)

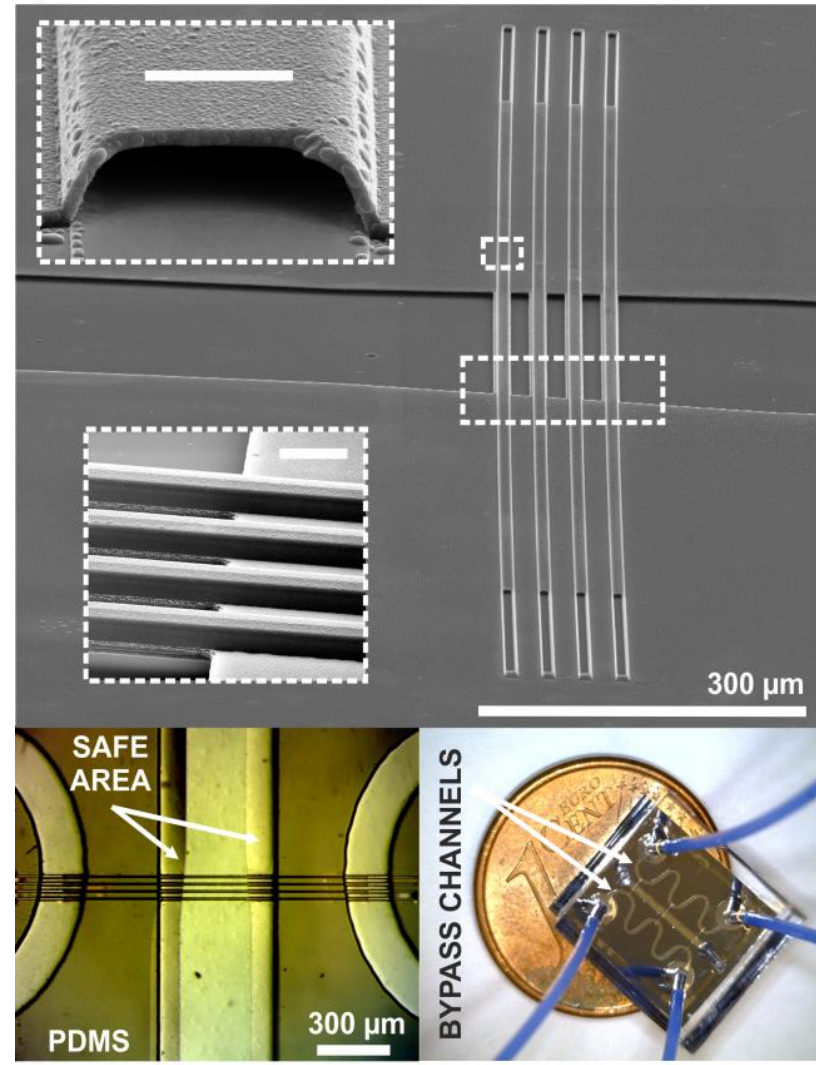

b)

c)

Fig. 2 a) Scanning electron microscope image of an array of four HMB resonators; inset 1 shows the cross section of an embedded microchannel of $20 \mu \mathrm{m} \times 4 \mu \mathrm{m}$ with $1 \mu \mathrm{m}$ thick polysilicon structural layer; inset 2 illustrates the $4 \mu \mathrm{m}$ topology and lateral distance of $13 \mu \mathrm{m}$ between the suspended array of resonators. Scale bar represents $10 \mu \mathrm{m}$. b) Optical microscope image illustrating the PDMS bypass channels connecting with the inlets of the HMB resonators. A safe area was set along the edges of the sensors $(50 \mu \mathrm{m})$ to avoid any leakage of uncured PDMS onto the structures. c) Chip with integrated microfluidics and external tubing connections for injection of sample solutions (dimensions $1 \mathrm{~cm} \times 1 \mathrm{~cm}$ ). 
1 dissolve the sacrificial layer. Also, in this step, the length of th 67 2 resonators was defined. Using HF $49 \%$, up to $1300 \mu \mathrm{m}$ long8 3 channels were emptied after approximately $25 \mathrm{~min}$ of isotropi 59 4 wet etching. The resonators were released in this procedure b 60 5 dissolving the $\mathrm{SiO}_{2}$ PECVD TEOS 2:1 beneath the polysilicon 1 6 microbeams at a lower etching rate than the BPSG sacrificia62

layer. Finally, the wafer was manually diced to have chips of 63 $8 \mathrm{~cm} \times 1 \mathrm{~cm}$. A scanning electron image of the final array of doubl 64 9 clamped beams is illustrated in Fig. 2a. Further details of devic65 10 fabrication are described in [27].

For injection of liquids inside the array of resonators, PDM\$7

12 bypass channels were replicated from a master mold of SU-88 13 negative photoresist (Microchem SU-8 2025). The master mol69 14 was fabricated by standard photolithography [28]. First, thæ0 15 photoresist was spun onto a silicon substrate to obtain a $35 \mu \mathrm{m} 1$ 16 thick layer and by soft-photolithography, the bypass channel\$2 17 were patterned. The $4 \mathrm{~mm}$ thick PDMS replica was prepared by 3 18 a 10:1 ratio of elastomer and curing agent from "Sylgard 1844 19 Silicon elastomer Kit". PDMS was cured in a hot plate at $80^{\circ} \mathrm{C5}$ 20 for $2 \mathrm{hrs}$. Thereafter, four through holes were perforated on each6 21 PDMS reservoir of the bypass channels with a biopsy punch7 22 (Harris Uni-CoreTM $1 \mathrm{~mm}$ I.D.) for external access of tubing8 23 connections and using a razor blade the PDMS was cut to hav 79 24 dimensions of $1 \mathrm{~cm} \times 1 \mathrm{~cm}$. Permanent bonding of PDMS to th80 25 devices was done by a stamp-and-stick technique in whic 1 26 uncured PDMS of 10:3 ratio was used as an adhesive [29182
Applying soft pressure to make the bonding process faste 3 resulted in reflow of the uncured polymer. Also, any increase ir 4 temperature caused clogging of the microchannels. Once th 85 uncured PDMS covered all over the surface, except those area86 corresponding to the microfluidic channels and the array $0 \$ 7$ resonators, the 10:3 PDMS was let to cure for $48 \mathrm{~h}$ at roons 8 temperature. This methodology yielded a good sealing of th89 topology of the structures and prevented any structural damage0 to the resonators as shown in Fig. 2b. The maximum pressure1 that this permanent union can withstand is up to $38 \mathrm{psi}$, which i\$2 adequate for flow rates below $100 \mu \mathrm{l} / \mathrm{min}$. Finally, four PEEK 3 tubes (Valco Instruments Co. Inc. JR-T-6009) of $250 \mu \mathrm{m}$ of i.d94 were affixed to each delivery channel reservoir. Fig. 205 illustrates the chip device with the integrated bypass channels. 96

\section{B. Experimental set-up}

Measurement of the HMB resonators was performed by 190 custom designed optical free-space interferometer. S1 fropl 1 supporting information shows a detailed scheme of opt 2 homebuilt experimental setup. A $632.8 \mathrm{~nm} \mathrm{HeNe}$ laser beam pfi 3 $1 \mathrm{~mm}$ diameter (JDSU 1101,1.5mW) passes through an opticaph isolator (Thorlabs IO-3D-633-VLP) to cancel undesired baqk5 reflections and noise fluctuations. A 50:50 beamsplittef6 (Thorlabs CM1-BS1) splits the laser beam by half to form 197 reference path, which reflects back from a reference mirror onfø8 a high-bandwidth photoreceiver (New Focus 1801). On theg active arm of the interferometer, the beam is expanded five time 0 with a beam expander (Thorlabs BE05M-A) for imaginq 1 purposes and for reducing the final spot size. The laser beam i\$ 2 focused tightly through a $20 \mathrm{x}$ microscope objective (Olymp $\$ \$ 3$ 20x, NA = 0.4) onto the middle point of the structures with the optical axis parallel to the bending motion of the resonators. The spot size of $1.3 \mu \mathrm{m}$ is calculated according to the Rayleigh criteria: $\omega_{0}=1.64 \lambda / 2 \mathrm{NA}$. Finally, the photoreceiver collects the interference pattern of the reflected light from a beam and the light from the reference mirror. All optical components of the experimental setup are rigidly assembled to an optical table with active mechanical isolation to compensate for undesired vibration drifts.

The actuation scheme of the array of resonators was done through a frequency sweep methodology that allowed a fast and high-throughput excitation over a range of frequencies around the central peak response of the resonators [30]. The excitation was achieved by means of a piezoceramic actuator (PImicos PIC181) placed beneath the chip, as Fig. 1 shows. The driven response of the resonators was acquired by a synchronization protocol between a function generator (Agilent 33220A) and a fast acquisition card (National Instruments PXI-5922) that avoided cross-talk interference between actuated and acquired signals. The function generator controlled the amplitude (1.5 Vpp) and sweep time (100 ms) of a sine-wave excitation signal over a $200 \mathrm{KHz}$ bandwidth around the central peak response of the resonators. Sequential evaluation of every resonator response was performed by the optical readout by transversally scanning each of the middle points of the beam resonators under the laser spot using an automated computer controlled 3-axis stage. Finally, we computed the Fast Fourier transform (FFT) of the acquired signals in LabVIEW to obtain the frequency spectrum response. The FFT was averaged three times to reduce noise fluctuations and a Lorentzian curve fitting algorithm computed in real-time the peak frequency response and Q-factor values.

The filling of embedded microchannels was done through an $\mathrm{H}$-shaped microfluidic configuration as Fig. $2 \mathrm{~b}$ shows. In each measurement, $250 \mu \mathrm{L}$ of sample volume was loaded and delivered into one of the bypass channels by a low-pressure valve (Valco Instruments Co. Inc. C22-3186) at a constant flow rate of $10 \mu \mathrm{L} / \mathrm{min}$ using a syringe pump (New Era Pump Systems Inc. NE-300). On the other bypass channel, a constant flow rate of 1 $\mu \mathrm{L} / \mathrm{min}$ rinsed the output of the microchannels continuously.

The calibration protocol of our sensor consisted of measuring the response of the resonators filled with air and water before determining the density and viscosity of samples. The response of the HMB devices was automatically captured by the optical readout interferometer while the resonators were actuated through the sweep frequency methodology. The temperature of the sensor was stabilized at $23^{\circ} \mathrm{C}$ by a closed loop temperature controller (Thorlabs T-Cube TEC Controller) with a resolution of $0.1^{\circ} \mathrm{C}$. Measurements were taken after $5 \mathrm{~min}$ of sample injection to ensure the liquid exchange inside the resonators and temperature stabilization. Then, the resonance frequency and the Q-factor were extracted from the resonance peak for each resonator. A calibration fit was done for the measurements of water and air. Before measuring a new sample fluid, the HMB resonators were cleaned with sodium dodecyl sulfate (SDS) hydrochloric acid $0.1 \mathrm{M}(\mathrm{HCl})$ and rinsed with plenty of water to reduce systematic instabilities on frequency. All measurements were done at least in triplicates. 


\section{RESUlTS AND DISCUSSION}

By considering polysilicon as structural material with density of $2331 \mathrm{~kg} / \mathrm{m}^{3}$ and Young Modulus of $160 \mathrm{GPa}$ [31], calculations from (3) showed that the expected fundamental resonant frequency of resonators $(\mathrm{L} \approx 275 \mu \mathrm{m})$ was approximately 918 $\mathrm{KHz}$ when filled with air. Instead, resonators showed a resonance frequency close to $650 \mathrm{KHz} \pm 10 \mathrm{~Hz}$ operating at $23^{\circ} \mathrm{C}$ and at atmospheric pressure conditions. This frequency variation was attributed to a modification of the effective length of the structures while releasing them from the substrate with the treatment of HF $49 \%$ acid during the fabrication process. By an optical characterization with microscope images, we measured a lateral over-etching distance of approximately $25 \mu \mathrm{m}$, which modified the final effective length of each beam. Based on calculations from (3), the resonant frequency of resonators with an over-length of $25 \mu \mathrm{m}$ at each clamped side was approximately of $651 \mathrm{KHz}$ when filled with air. An additional photolithographic mask to empty and release the resonators in two consecutive steps can further reduce the lateral over-etching effect.

After characterizing the resonators in the unfilled state, we studied the performance of the sensors when filled with water. The array of beams exhibited a frequency response close to 502 $\mathrm{KHz} \pm 11 \mathrm{~Hz}$, which represents a frequency shift of $148 \mathrm{kHz}$ $(22.77 \%)$ with respect to their response in air. In the Electronic Supplementary Information (ESI) we show a video of the fluid exchange inside the HMB device. The filling capabilities of the embedded microchannels were successfully proven owing to the physical properties of the internal sidewalls, which presented low porosity and a high planarization level. The frequency shift of the resonators was in accordance with the change in density of the fluid contained inside the microfluidic channels. Sensor noise was estimated based on the evaluation of the standard deviation of the frequency response of the resonators when fillegु with water, specifically when no frequency variation waş8 expected $\left(\Delta f_{R}=0\right)$. Over a period of $45 \mathrm{~min}$, the resonanf9 frequency peak showed a standard deviation of $\sigma_{R}= \pm 10 \mathrm{~Hz}_{60}$ Although random sources of noise were present in th 81 acquisition system, such as laser amplitude fluctuations and 82 variations of the optical focusing of resonators, other sources of 3 error could potentially be controlled. For example, by increasing 4 the number of acquired samples and by reducing the excitations frequency bandwidth, the systematic errors introduced by the 66 Lorentzian curve fitting of the frequency response were 67 minimized.

Thereafter, to calculate the mass responsivity of the device 68 we used four samples with different and well-known densities (from Sigma Aldrich): diethyl ether $\left(713.4 \mathrm{~kg} / \mathrm{m}^{3}\right)$, isopropanol $\left(786 \mathrm{~kg} / \mathrm{m}^{3}\right)$, ethanol $\left(789 \mathrm{~kg} / \mathrm{m}^{3}\right)$, and acetic acid $\left(1049 \mathrm{~kg} / \mathrm{m}^{3}\right)_{72}$ The latter one was chosen to measure the linear response of the sensor beyond the density of the reference liquid (water). Fig. 34 shows the frequency response depicted by these sample solutions 5 with respect to their reference density values for a single resonator. The results demonstrated a clear relationship between the measured resonant frequency peak shift and the sample solution density, according to the model proposed by (3). To determine the sensitivity of the sensor, a linear curve fitting of

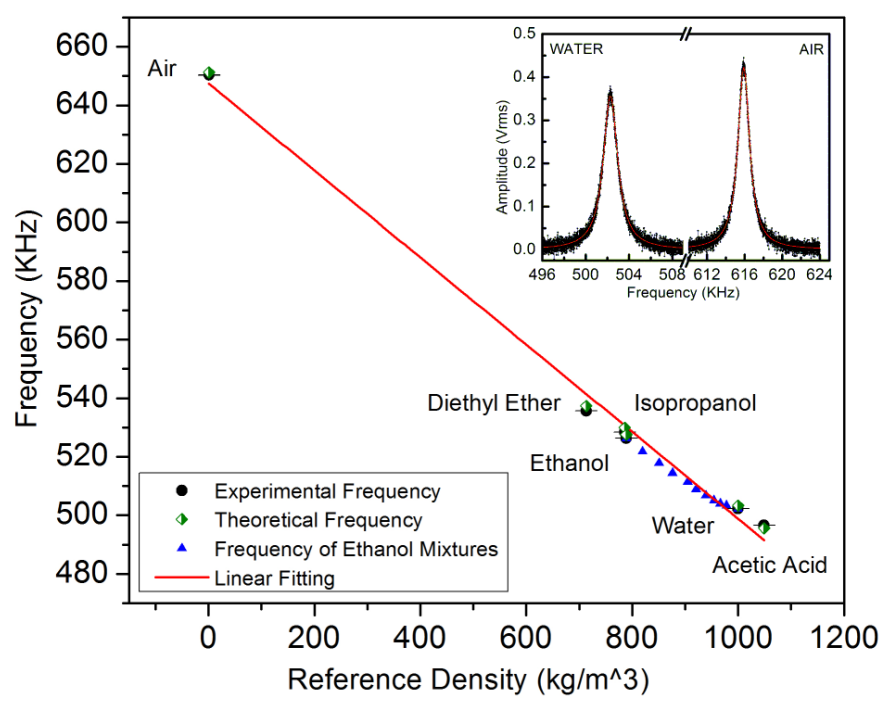

Fig. 3 Resonant frequency response of a resonator when filled with different solutions with well-known densities, including water/ethanol mixtures (blue triangle markers). The inset shows the peak frequency response of the resonator before and after filling it with water indicating a frequency shift of $148 \mathrm{KHz}$ with a $41.1 \%$ quality factor decay. Error bars are smaller than black dots and represent a standard deviation of $\pm 10 \mathrm{~Hz}$.

TABLE I

PERFORMANCES OF THE ARRAY OF HMB RESONATORS

\begin{tabular}{lllll}
\hline \hline & & $\begin{array}{l}\text { Resonance } \\
\text { frequency } \\
\mathrm{f}_{0}(\mathrm{kHz})\end{array}$ & $\begin{array}{l}\text { Extracted } \\
\text { slope }\left(\mathrm{S}_{\mathrm{A}}\right)\end{array}$ & $\begin{array}{l}\text { Experimental } \\
\text { mass } \\
\text { responsivity } \\
\mathrm{Hz} / \mathrm{kgm}^{-3}\end{array}$ \\
Bridge & $\begin{array}{l}\text { Sensor } \\
\text { mass }(\mathrm{ng})\end{array}$ & in air & 147.77 & 7.38 \\
B1 & 17.69 & 650.119 & 147.86 & 7.39 \\
B2 & 17.30 & 650.422 & 148.19 & 7.40 \\
B3 & 16.90 & 651.094 & 148.75 & 7.43 \\
B4 & 16.50 & 651.262 & & \\
\hline \hline
\end{tabular}

data within this range of densities was calculated for each HMB resonator, as Table 1 shows. The mass responsivity was obtained by dividing the sensitivity with respect to the volume of a single embedded microchannel $(\sim 20 \mathrm{pL})$. The results show a better mass responsivity than that achieved by two previous SMR approaches [32],[13]. However, the mass responsivity of HMB resonators is surpassed by that of SMR devices with smaller effective mass [19],[20]. Also, our findings demonstrated that a minimum resolvable density change of $0.068 \mathrm{~kg} / \mathrm{m}^{3}$ was achieved for a frequency resolution of $\pm 10 \mathrm{~Hz}$.

Owing that the mass responsivity of the array of $\mathrm{HMB}$ resonators resulted quite similar $(\sim 7.4 \mathrm{~Hz} / \mathrm{pg})$, the average response of the four sensors was calculated in order to increase the throughput in the analysis of the following samples. To study the sensor performance as a rheology analyzer we prepared binary solutions of ethanol and water. We injected solutions of ethanol with concentrations in volume from $0 \%$ to $100 \%$, with increments of $10 \%$. Besides, the density of ethanol/water mixtures was calculated with a commercial pycnometer $(10 \mathrm{ml}$ pycnometer, Brand) at a fixed temperature of $23^{\circ} \mathrm{C}$ to evaluate the frequency response of the sensor as a function of the density of the samples. Fig. 4a shows the average frequency peak measurement over a time period of $10 \mathrm{~min}$ for each ethanol concentration. As expected, the interplay between the frequency 
a)

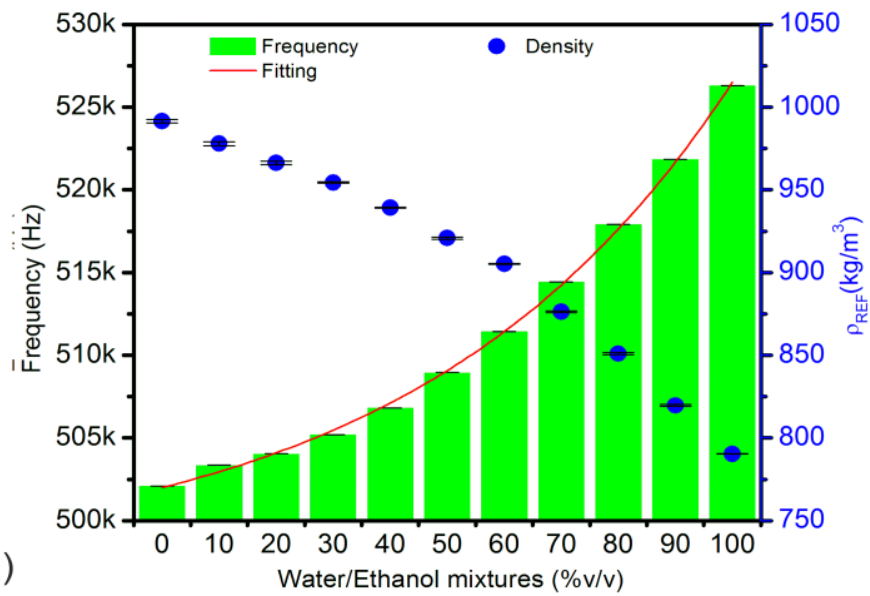

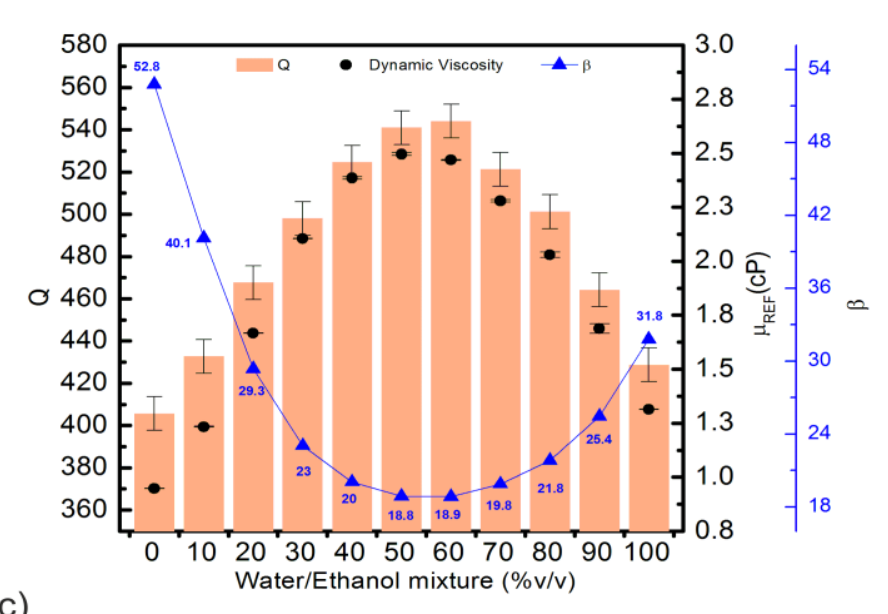

c)

d) b)

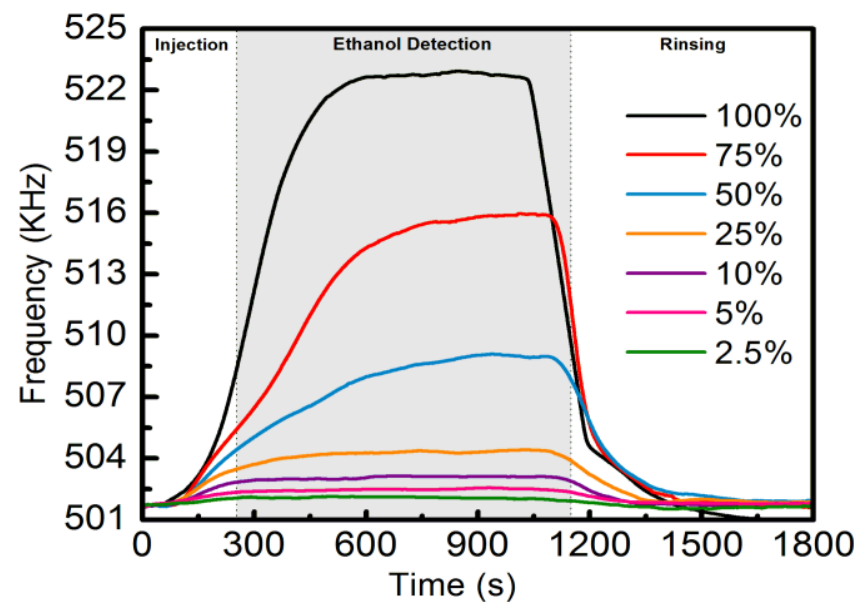

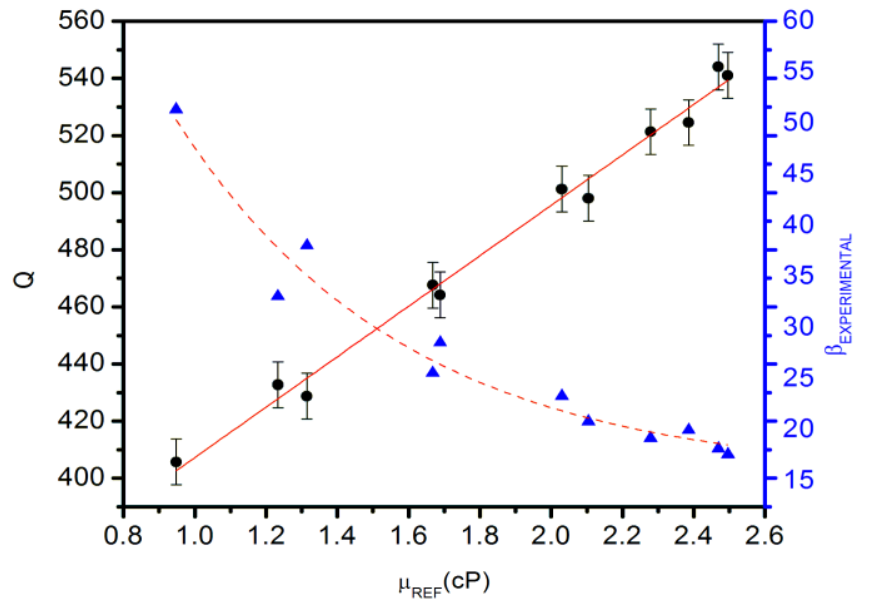

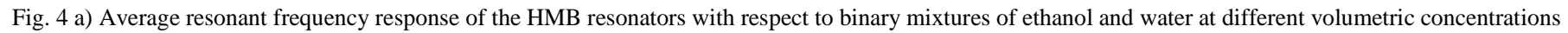

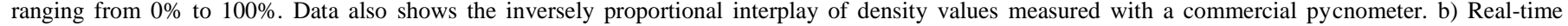

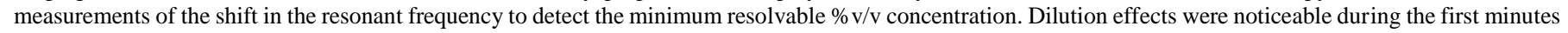

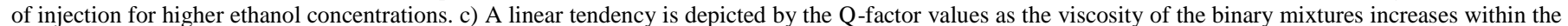
viscosity regime from $1 \mathrm{cP}$ to $2.6 \mathrm{cP}$. d) The experimental values for $\beta$ demonstrate de interplay between fluid inertia and Q-factor values.

1 of the sensor and the density was inversely proportional witbo 2 respect to the content of water/ethanol mixtures. This tendenc21 3 was also observed in other calibration protocols [33]. The lineaß2

curve fitting of the frequency with respect to the density within 3 this range showed a slope of $146 \mathrm{~Hz} / \mathrm{kgm}^{-3}$. As Fig. 4b shows, a4 complete sample exchange inside of resonators took about $\mathbf{Z} 5$ minutes for a $10 \mu \mathrm{L} / \mathrm{min}$ flow rate in the inlet bypass channeh 6 We note that this time could be reduced by controlling the inlet 7 bypass channel inflow to diminish dilution effects of mixture 8 with the initial reference liquid (water) that was contained inside9 of resonators. Furthermore, the results demonstrated ab0 increasing exponential trend in frequency as the ethanof 1 concentration increased while modifying the sample density 32 according to the following curve fitting equation:

$$
f(\% v / v)=497178.74+4824.114 e^{-0.01806(\% v / v)}
$$

From here, the minimum resolvable ethanol concentration of 7 $0.115 \%$ was computed for a frequency resolution of $\sigma_{R}= \pm 1 \hat{\theta}^{8}$
Hz. Sensor Signal-to-Noise ratio (SNR) was calculated for the minimum ethanol concentration as $\Delta \mathrm{f}_{2.5 \%} / \sigma_{\mathrm{R}}$; where $\Delta \mathrm{f}_{2.5 \%}=418$ $\mathrm{Hz}$ is the frequency shift derived from a $2.5 \%$ ethanol concentration, as shown in Fig. 4b, yielding a SNR of 41.8 .

As regards the energy dissipation of the HMB device, the reported Q-factor value of resonators was $692 \pm 10$ in air. However, after filling the microfluidic channels with water, the Q-factor decreased to $405.70 \pm 10$ for all the resonators, which represents a decay of $41.1 \%$. This effect is similar to another approach of SMR devices [24] when the air was replaced with water showing a $40 \%$ decay of the quality factor. To better understand this behavior, we compared the average response of the quality factors of the array of resonators when filled with binary mixtures of water/ethanol, with the corresponding dynamic viscosities. The viscosities were calculated with a commercial Ubbelohde viscometer (UBBEL02UKC, SigmaAldrich) at a fixed temperature of $23^{\circ} \mathrm{C}$, as Fig. $4 \mathrm{c}$ shows. Interestingly, the experimental results showed a decrease in the dissipation of energy of the resonators (enhancement of quality 
1 factor) as a function of increasing viscosity. This effect is

2 contrary to the Q-factor response depicted by low-stress silicon 3 nitride SMR devices [13] in which the quality factor decreased 4 as a function of increasing viscosity of water/ethanol mixtures. 5 This can be explained in terms of the dimensionless frequency 6 number $\beta$ by considering the height of our microfluidic channels

$7 \quad\left(\mathrm{~h}_{\text {fluid }}=4 \mu \mathrm{m}\right.$ ) and taking into account the values of density and 8 viscosity calculated from the reference sensors. For instance, the 9 value of $\beta$ after filling the microchannel with ethanol was approximately 31.81 . Fig. $4 \mathrm{c}$ shows that for our device, the computed values of $\beta$ for water/ethanol mixtures are within the low inertia regime $(\beta<46)$. In this regime, since there is little inertia, the fluid follows the solid displacement of the resonator resembling a rigid-body oscillation. This explains the improvement in Q-factor values with increasing viscosity from $1 \mathrm{cP}$ to $2.6 \mathrm{cP}$. From here, we correlated the Q-factor values of water/ethanol samples with respect to their reference viscosity values as shown in Fig. 4d, which resulted in a empirical linear behavior for the water/ethanol mixtures according to the following curve fitting approximation:

$$
Q(\mu)=325.78+82.5(\mu)
$$

The non-monotonic interplay between the water/ethanol samples and the Q-factor, along with the polar nature of the ethanol and water molecules indicate that (6) is applicable within a short range of viscosities. From here, the minimum resolvable viscosity change of $0.15 \mathrm{cP}$ was computed for a Q-factor resolution of \pm 10 . Experimentally, $\beta$ decreased exponentially as a function of sample viscosity, which confirms the low-inertia regime of the sensor, as Fig. 4d shows.

Finally, we tested the device performance to measure the density and viscosity of a variety of alcoholic beverages. We compared four distilled beverages that contained no added sugar (spirits), and one distilled beverage with added sugar and flavorings. For the group of distilled alcoholic beverages, there was a close correlation as density increased with a percentage error below $0.56 \%$. In particular, beverages such as vodka, whisky and rum, which contain $40 \%$ of Alcohol by Volume (ABV), depicted close frequency values among them with an average resonant frequency of $506.6 \mathrm{KHz} \pm 11 \mathrm{~Hz}$, as Fig. $5 \mathrm{a}$ shows. However, measurements with coffee liquor exhibited a frequency response $(496.105 \mathrm{KHz} \pm 11 \mathrm{~Hz})$ below the frequency response of water, showing that sugar concentration and flavorings influence density values more than the ethanol content. In comparison with the time used by $\mathrm{U}$-tube viscometers $(\sim 15 \mathrm{~min})$ and pycnometers ( $\sim 2 \mathrm{~min}$ ) to determine the viscosity and density of alcoholic drinks, the HMB device significantly reduced the time for calculating both parameters. In a single measurement both the viscosity and density of alcoholic drinks were determined with our methodology in 7 min requiring sample volumes of only 250 $\mu \mathrm{l}$. Afterwards, we calculated by (6) the dynamic viscosity of the alcoholic drinks by tracking the response of the Q-factor of the sensor (see Fig. 5b). We compared the calculated dynamic viscosity of each alcoholic drink with their corresponding dynamic viscosity obtained using the U-tube sensor. The results showed a percentage error below $2 \%$. Deviations from the
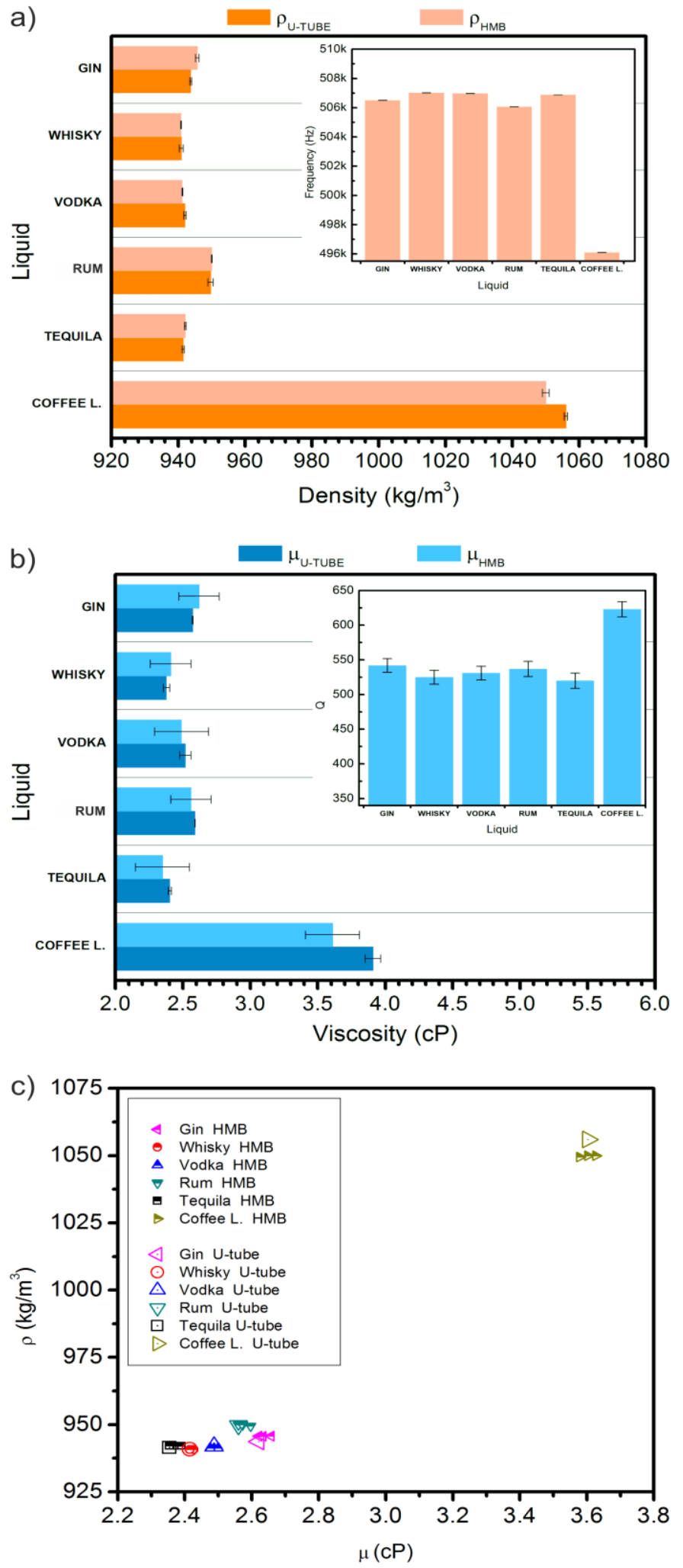

Fig. 5 a) Resonant frequency response of various alcoholic drinks. b) Calculated viscosity of distilled alcoholic drinks (the error increases for viscosities beyond $2.6 \mathrm{cP}$ as is the case for the coffee liquor). c) Comparison of experimental values from the HMB sensor with respect to the values from reference sensors for identification of alcoholic drinks. 
1 expected value (7.67\%) were observed for the coffee liquos7 2 beverage given that its dynamic viscosity is out of the linears 3 viscosity regime of the HMB sensor. Error bars of the HMB9 4 viscosity measurements in Fig. 5b make challenging th 60 5 identification of individual alcoholic beverages with close $A B b_{1}$

\section{percentages. The accuracy of the dynamic viscosit 2 measurements can be improved by operating the sensors in 83 moderate vacuum environment. Identification of alcoholiq drinks with the HMB devices is only feasible as a function of both the obtained density and dynamic viscosity as Fig. 585 shows.}

Regarding the material properties of the sensor, a structura 66 material such as polysilicon can be easily oxidized by differen ${ }^{7}$ methods such as thermal oxidation or by direct streaming o甲 8 oxidation agents. This advantage is of importance in order t 69 modify the hydrophilic properties of the inner microfluidic 0 walls to facilitate the filling of resonators with fluids of different viscosities. Another advantage of polysilicon is the reduced time 1 for etching the sacrificial layer in only a few minutes.

\section{SUMMARY AND CONCLUSIONS}

We have validated the fabrication and performance of an array 8 of polysilicon doubly clamped resonators with embedded9 microfluidic channels to work as a mass density and viscosit 80 sensor. The fabrication of the devices was accomplished using 82 standard surface micromachining techniques. The doubl 83 clamped configuration of the resonators provided mor 84 flexibility for fabricating straight microchannels with differen 85 dimensions to facilitate the input and output of liquid phas87 solutions and also to reduce the clogging of the microfluidi 88 channels.

We have achieved a proper on-chip integration of polyme based channels using a permanent bonding strategy with PDMS92 This included the sealing with good step coverage of 3 microstructures with features that protruded out from the base 95 substrate. On the other hand, the implemented optical readout 6 granted the efficient acquisition of the nanometric out-of-plang 98 displacements of the HMB devices automatically. Enhancing the9 frequency resolution of the detection system by implementin 60 parametric feedback oscillator methodologies and operating the1 devices in a moderate vacuum can continuously improve the3 sensitivity and resolution of HMB devices at least one order $\mathbf{b 0 4}$ magnitude as it has been demonstrated by a previous approaet 5 [34].

The experimental results demonstrated that the system coul 08 be used for high-throughput measurement of liquid phase 9 analytes. We have experimentally proven the linearity betwe 110 the resonance frequency shifts of the resonators and the liquid 2 density, when testing different samples ranging from solvents 13 organic solutions to alcoholic beverages, streamed through thi 14 embedded microchannels in real-time. Furthermore, we found 46 correlation of the viscosity of the samples as a function bf7 increasing the Q-factor value of the resonators. Due to the no 118 monotonic energy dissipation of the HMB device, sample0 viscosity measurements were feasible in a short linear regimme2, 1 between $1 \mathrm{cP}$ to $2.6 \mathrm{cP}$, with a resolution of $0.15 \mathrm{cP}$. Importantl 122 the array of HMB resonators could characterize low volumes of liquids at atmospheric pressure conditions with a better mass responsivity $(7.4 \mathrm{~Hz} / \mathrm{pg})$ than current SMR devices [13] and with a comparable response to another competitive approach [32]. Thus, this work is a step towards the development of a multiplexed platform capable of rapid monitoring of rheological properties of distinct fluid samples.

\section{ACKNOWLEDGMENT}

Authors acknowledge the support from National Council for Science and Technology (CONACyT-Mexico). We are also thankful to V. Solis-Tinoco for her collaboration and discussions in the microfluidic integration of devices.

\section{REFERENCES}

[1] R. Paxman, J. Stinson, A. Dejardin, R. A. McKendry, and B. W. Hoogenboom, "Using Micromechanical Resonators to Measure Rheological Properties and Alcohol Content of Model Solutions and Commercial Beverages," Sensors, vol. 12, no. 5, pp. 6497-6507, 2012.

[2] J. F. Vélez-Ruiz and G. V. Barbosa-Cánovas, "Rheological properties of concentrated milk as a function of concentration, temperature and storage time,” J. Food Eng., vol. 35, no. 2, pp. 177-190, 1998.

[3] P. Rust, D. Cereghetti, and J. Dual, "A micro-liter viscosity and density sensor for the rheological characterization of DNA solutions in the kilohertz range," Lab. Chip, vol. 13, no. 24, pp. 4794-4799, 2013.

[4] M. Godin, A. K. Bryan, T. P. Burg, K. Babcock, and S. R. Manalis, "Measuring the mass, density, and size of particles and cells using a suspended microchannel resonator," Appl. Phys. Lett., vol. 91, no. 12, pp. 123121-123121-3, 2007.

[5] J. Zhang, C. Dai, X. Su, and S. J. O'Shea, "Determination of liquid density with a low frequency mechanical sensor based on quartz tuning fork," Sens. Actuators B Chem., vol. 84, no. 2-3, pp. 123-128, 2002.

[6] P. Enoksson, G. Stemme, and E. Stemme, "Fluid density sensor based on resonance vibration," Sens. Actuators Phys., vol. 47, no. 1-3, pp. 327$331,1995$.

[7] I. Etchart et al., "MEMS sensors for density-viscosity sensing in a lowflow microfluidic environment," Sens. Actuators Phys., vol. 141, no. 2, pp. 266-275, 2008.

[8] B. A. Bircher et al., "Real-Time Viscosity and Mass Density Sensors Requiring Microliter Sample Volume Based on Nanomechanical Resonators," Anal. Chem., vol. 85, no. 18, pp. 8676-8683, 2013.

[9] L. Müller et al., "Investigation of Prothrombin Time in Human WholeBlood Samples with a Quartz Crystal Biosensor," Anal. Chem., vol. 82, no. 2, pp. 658-663, 2010.

[10] O. Cakmak et al., "Microcantilever based disposable viscosity sensor for serum and blood plasma measurements," Methods, vol. 63, no. 3, pp. 225232, 2013.

[11] M. Youssry, N. Belmiloud, B. Caillard, C. Ayela, C. Pellet, and I. Dufour, "A straightforward determination of fluid viscosity and density using microcantilevers: From experimental data to analytical expressions," Sens. Actuators Phys., vol. 172, no. 1, pp. 40-46, 2011.

[12] J. Toledo et al., "Comparison of quartz tuning forks and AlN-based extensional microresonators for viscosity measurements in oil/fuel mixtures," in Proc. SPIE 8763, Smart Sensors, Actuators, and MEMS VI, 2013, p. 87630L.

[13] M. F. Khan et al., "Online measurement of mass density and viscosity of pL fluid samples with suspended microchannel resonator," Sens. Actuators B Chem., vol. 185, pp. 456-461, 2013.

[14] S. Boskovic, J. W. M. Chon, P. Mulvaney, and J. E. Sader, "Rheological measurements using microcantilevers," J. Rheol. 1978-Present, vol. 46, no. 4, pp. 891-899, 2002.

[15] N. McLoughlin, S. L. Lee, and G. Hähner, "Temperature dependence of viscosity and density of viscous liquids determined from thermal noise spectra of uncalibrated atomic force microscope cantilevers," Lab. Chip, vol. 7, no. 8, p. 1057, 2007.

[16] M. Hennemeyer, S. Burghardt, and R. W. Stark, "Cantilever Microrheometer for the Characterization of Sugar Solutions," Sensors, vol. 8, 


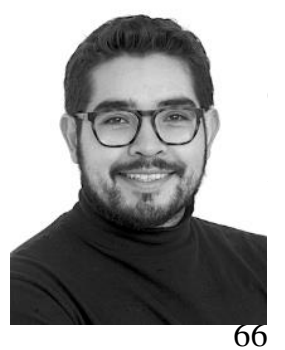

Salomón Marquez received his MSc degree in Electrical Engineering from The Center for Research and Advanced Studies of the National Polytechnic Institute (CINVESTAV-Mexico) back in 2010. In 2016, he obtained his Ph.D. in electronics engineering working at the Catalan Institute of Nanoscience and

Nanotechnology (ICN2, CSIC) in the
Bioanalytical Applications groun

no. 1, pp. 10-22, 2008. of density and viscosity of liquids based on resonance curves of
uncalibrated microcantilevers," Appl. Phys. Lett., vol. 89, no. 18, 70 184106, 2006.

9] V. Agache, G. Blanco-Gomez, F. Baleras, and P. Caillat, "An embedded 4 microchannel in a MEMS plate resonator for ultrasensitive mass sensing5 in liquid," Lab. Chip, vol. 11, no. 15, pp. 2598-2603, 2011. R. A. Barton, B. Mlic, S. S. Verbridge, B. R. Cipriany, J. M. Parpia, and 77 G. Craighead, "Fabrication of a Nanomechanical Mass Sensor Containing 8

21] J. L. Arlett and M. L. Roukes, "Ultimate and practical limits of fluid-base 79 mass detection with suspended microchannel resonators," J. Appl. Phys80 vol. 108, no. 8, pp. 84701-84701-11, 2010. Vibrating Beams Using Four Engineering Theories," J. Sound Vib., vol. 225, no. 5, pp. 935-988, 1999.

23] N. Lobontiu, Mechanical Design of Microresonators: Modeling and Applications, 1 edition. New York: McGraw-Hill Professional, 2005. 22, p. 228103, 2009 2010.

27] S. Marquez, M. Alvarez, D. Farina, C. Dominguez, and L. M. Lechug9, "Towards a biosensing multiple platform based on an array of hollow2 microbridge resonators," in 2014 IEEE SENSORS, 2014, pp. 329-331. 93 D. Qin, Y. Xia, and G. M. Whitesides, "Rapid prototyping of complex
structures with feature sizes larger than $20 \mu \mathrm{m}$," Adv. Mater., vol. 8, no. 11, pp. 917-919, 1996. strates for monolithic microfluidic applications," b7 Micromechanics Microengineering, vol. 24, no. 7, p. 75010, 2014. "Fast frequency sweeping in resonance-tracking SPM for high-resolutip00 AFAM and PFM imaging," Nanotechnology, vol. 23, no. 49, p. 4957951 2012.

32] J. Lee et al., "Suspended microchannel resonators with piezoresistive sensors," Lab Chip, vol. 11, no. 4, pp. 645-651, 2010.

33] I. S. Khattab, F. Bandarkar, M. A. A. Fakhree, and A. Jouyban, "Density, viscosity, and surface tension of water+ethanol mixtures from 293 to 323K," Korean J. Chem. Eng., vol. 29, no. 6, pp. 812-817, 2012.

S. Olcum et al., "Weighing nanoparticles in solution at the attogram scale,” Proc. Natl. Acad. Sci., p. 201318602, 2014. developing MEMs/NEMs technology with embedded microchannels for biosensing applications. His fields of interest include design and modeling of MEMs devices, integration of microfluidic applications and development of biomedical sensors.

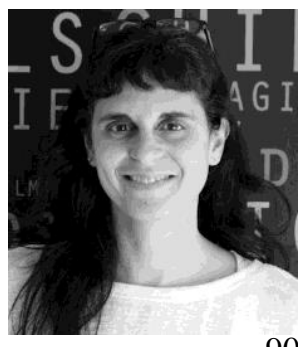

Mar Álvarez is currently a researcher at the Biomedical Applications Group at the Institute of Microelectronics of Barcelona of the Spanish National Research Council (IMB-CNM, CSIC). Her main research interest is focused on the technological development of microfluidics and biosensor devices and

90 its integration on lab-on-a-chip and organ-on-chip platforms for the performance of a wide range of biomedical applications. She obtained her MSc and PhD degrees in Physics from the Autonoma University of Madrid, Spain.

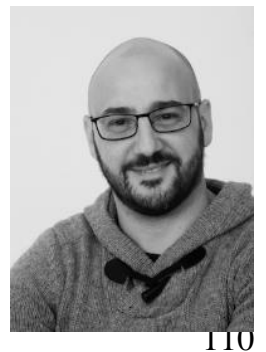

David Fariña Santana is a researcher at the Nanobiosensors and Bioanalytical Applications group and the Catalan Institute of Nanoscience and Nanotechnology (ICN2), with a special interest in software and hardware development for optical biosensors and their integration in lab-onchip platforms. He obtained the ME degree and the $\mathrm{PhD}$ in Telecommunications 111 Engineering at the University of Las Palmas de Gran Canaria, 112 Spain.

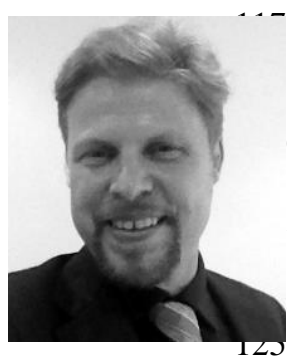

Antoni Homs-Corbera is, since March 2015, a researcher at the Catalan Institute of Nanoscience and Nanotechnology (ICN2). He graduated from the Universitat Politècnica de Catalunya (UPC, ES) in Telecommunications and Electronics Engineering, back in 1999. Later, he received the degrees of Master in Biomedicine, from the Universitat de Barcelona (UB, Barcelona, ES), and Master in Nanomaterials, from the Imperial College (London, UK). He was awarded his 
$\mathrm{PhD}$ in Biomedical Engineering from the UPC in 2005. He als61 holds an MBA for international executives (GEMBA) by IES162 since 2013. During his career, he has worked in both privat63 industry and public research, holding positions in severa 4 institutions across the globe. His research mainly focusses on th65 biomedical use of micro and nanotechnologies, BioMEMS an 66 Lab-on-a-Chip devices.

16 Carlos Dominguez received the B.S., M.S., and Ph.D. degrees in chemistry from the Universidad Complutense of Madrid, Spain, in 1980 , and 1985 , respectively. He became a member of the scientific staff at the Instituto de Microelectrónica de Barcelona (IMB-CNM, CSIC) in 1986. Since 1991 he has been a Full Professor. He is involved in materials and process development for new

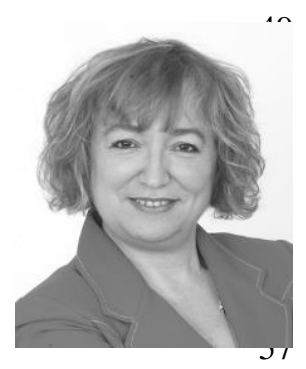

Laura M. Lechuga is Full Professor at the Catalan Institute of Nanoscience and Nanotechnology (ICN2), Spanish National Research Council (CSIC) \& CIBER-BBN. Since 2013 she is a Distinguished Visiting professor (PVE) at the Dept. of Microwaves and Photonics, School of Electrical and Computer Sciences, University of Campinas (Brazil) and Fellow of the Optical Society (OSA) since 2014. The principal focus of her research program is the technological development of photonic (plasmonics and silicon-based) biosensors, their integration in portable lab-on-a-chip platforms and their application for clinical and environmental diagnostics. She has published over 200 articles, book chapters and proceedings, has 8 families of awarded patents at European, US or international level,and has presented her work in more than 300 invited talks. She was the driving force for the establishment of one spin-off company in 2004 (SENSIA, S.L.) and co-founder of another spin-off in 2010 (BIOD, S.L.). Prof. Laura Lechuga is associate editor of the IEEE Photonics Journal, associate editor of the J. Optics and Laser Technology (Elsevier). 